\title{
Second-harmonic double-resonance cones in dispersive hyperbolic metamaterials
}

\author{
Domenico de Ceglia,,${ }^{1, *}$ Maria Antonietta Vincenti, ${ }^{1}$ Salvatore Campione, ${ }^{2}$ Filippo Capolino, ${ }^{2}$ \\ Joseph W. Haus, ${ }^{1,3}$ and Michael Scalora ${ }^{4}$ \\ ${ }^{1}$ National Research Council-AMRDEC, Charles M. Bowden Research Laboratory, Redstone Arsenal, Alabama 35898, USA \\ ${ }^{2}$ Department of Electrical Engineering and Computer Science, University of California Irvine, California 92697, USA \\ ${ }^{3}$ Electro-Optics Program, University of Dayton, 300 College Park, Dayton, Ohio 45469, USA \\ ${ }^{4}$ Charles M. Bowden Research Laboratory, AMRDEC, U.S. Army RDECOM, Redstone Arsenal, Alabama 35898, USA
}

(Received 23 May 2013; published 19 February 2014)

\begin{abstract}
We study the formation of second-harmonic double-resonance cones in hyperbolic metamaterials. An electric dipole on the surface of the structure induces second-harmonic light to propagate into two distinct volume plasmon-polariton channels: a signal that propagates within its own peculiar resonance cone and a phase-locked signal that is trapped under the pump's resonance cone. Metamaterial dispersion and birefringence induce a large angular divergence between the two volume plasmon polaritons, making these structures suitable for subwavelength second- and higher-harmonic imaging microscopy.
\end{abstract}

DOI: 10.1103/PhysRevB.89.075123

PACS number(s): 42.65.Ky, 41.20.Jb, 68.65.Ac, 78.20.-e

\section{INTRODUCTION}

The inhomogeneous or phase-locked solution of the nonlinear Helmholtz equation for harmonic generation processes $[1,2]$ travels at the same phase and group velocity as the fundamental frequency (FF) signal $[3,4]$. It has also been shown that the generated phase-locked components survive in the presence of linear absorption at the harmonic frequencies [5]. In this paper, we predict that a hyperbolic metamaterial supports resonance cones (RCs), or volume plasmon polaritons, in the presence of absorption at the fundamental and harmonic frequencies, propagating in directions that are tilted with respect to the optical axis of the metamaterial.

Recently, hyperbolic metamaterials have attracted a great deal of attention for their unique dispersive properties. We refer the reader to Refs. [6-8] for recent reviews on the topic. Hyperbolic metamaterials may be implemented in a layered metal-dielectric stack configuration. In these environments, the study of harmonic generation necessitates the inclusion of actual material dispersion and absorption. Additionally, the artificial birefringence found in this class of metamaterials can support the real parts of the ordinary and extraordinary effective permittivities with opposite signs, leading to a hyperbolic dispersion relation [9-11] and the propagation of large but finite spatial frequency components $[12,13]$. In contrast, in the effective-medium approximation, there is no upper bound to the maximum spectral component allowed in the medium. Multilayered media increase the efficiency of nonlinear optical effects via phase matching or local field enhancement (Ref. [14] and references therein). It has been shown that the further inclusion of cubic nonlinearities can improve the quality of subwavelength imaging by inhibiting diffraction via self-focusing while maintaining broadband operation and increased propagation distances compared to linear metamaterials [15]. Favorable conditions for imaging through flat hyperbolic metamaterial lenses [16] are met when the ordinary effective permittivity $\varepsilon_{0}$, i.e., the permittivity component parallel to the metal-dielectric interfaces vanishes

*domenico.deceglia@us.army.mil
$[17,18]$. Under these circumstances, the field distribution of an object at the input surface of the multilayer lens is imaged at the output plane with minimal distortion thanks to the formation of parallel rays that carry high spatial frequency components along the optical axis $(z$ axis, direction normal to the interfaces). Subwavelength imaging in metal-dielectric stacks can be further improved in the superguiding $[19,20]$ and canalization [21,22] regimes as described in Ref. [23] in terms of the point spread function. The introduction of gain in metal-dielectric stacks has also been shown to effectively improve super-resolution by compensating metal losses [24]. The multilayer focusing capabilities [19] may be tailored by properly designing the curvature of the dispersion relation [25]. Subwavelength imaging can also be attained in other extremely anisotropic configurations, such as wire metamaterials [26].

The chromatic dispersion of the metal permittivity causes $\varepsilon_{\mathrm{o}} \approx 0$ only in a narrow wavelength range around $\lambda_{0}$. Increasing the optical path length in the dielectric layers pushes $\lambda_{0}$ from the near UV to the visible and infrared regions. For a given configuration, when $\lambda>\lambda_{0}$, the multilayer displays negative ordinary effective permittivity, whereas, the extraordinary effective permittivity $\varepsilon_{\mathrm{e}}$, i.e., the permittivity along the optical axis, is positive. In this regime, the stack still allows propagation of large spectral wave numbers but not along the optical axis direction. Instead, the slab supports RCs [27] or volume plasmon polaritons [28] propagating in a preferred direction [29] tilted at some angle with respect to the optical axis.

Subwavelength interference of volume plasmon polaritons in a hyperbolic metamaterial substrate has been observed in a Young's double slit experiment [28]. It has been suggested that RCs may be used effectively to design single-photon sources [30], but so far, only the linear properties have been investigated. This notwithstanding, we will show that nonlinear optical interactions in these metamaterials may lead to exotic phenomena with the potential for subwavelength imaging applications as detailed in the representative case described in Sec. IV where we investigate second-harmonic (SH) field patterns generated by subwavelength objects located on the surface of nonlinear anisotropic slabs. While the FF signal diffracts in a RC with a single volume plasmon polariton, 
in Sec. II we predict that the diffraction of harmonic signals is channeled mainly in two distinct volume plasmons: a homogeneous volume plasmon polariton that propagates in the $\mathrm{RC}$ of the harmonic frequency signal and a phase-locked volume plasmon polariton, which is trapped in the RC of the FF signal. In Sec. III, we then highlight the advantage of using a simple effective homogenized model of hyperbolic metamaterials as well as the differences with the Bloch theory model that accounts for the lamellar discreteness. Finally, in Sec. IV, we give an example of super-resolution for $\mathrm{SH}$ light generated by nonlinear and subwavelength objects placed on the surface of a metal-dielectric hyperbolic metamaterial. In the proposed configuration, we exploit the homogeneous volume plasmon polariton of the SH signal through which we are able to discriminate the presence of two objects with subwavelength separation.

\section{FORMATION OF SECOND-HARMONIC DOUBLE-RESONANCE CONES}

We consider a hyperbolic metamaterial as in Fig. 1(a) made by alternating 5-nm-thick planar layers of silver and a generic dielectric medium having $\varepsilon_{\mathrm{d}}=4$, compatible, for example, with metal oxides, such as $\mathrm{Ta}_{2} \mathrm{O}_{5}, \mathrm{TiO}_{2}$, and $\mathrm{ZnO}$, and with $\mathrm{LiNbO}_{3}$ and $\mathrm{SrTiO}_{3}$. The complex permittivity of bulk silver $\varepsilon_{\mathrm{m}}$, accounting for losses, is taken from Palik's handbook [31]. The metal-dielectric interfaces lie on the $x-y$ plane. By adopting a quasistatic and local effectivemedium approximation, a simplified homogenization of this lamellar structure results in a uniaxial anisotropic model for
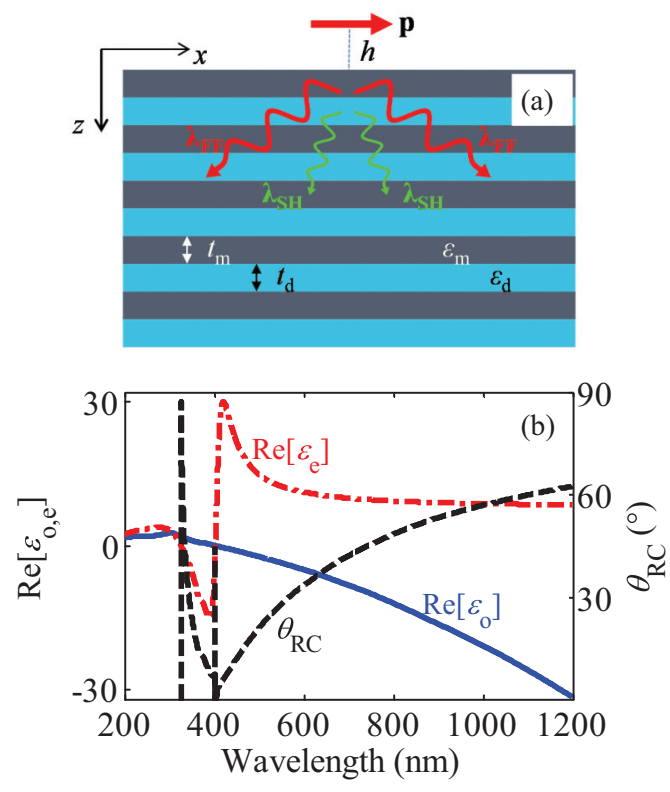

FIG. 1. (Color online) (a) Schematic of an electric line dipole $\mathbf{p}$ on the surface of a hyperbolic metamaterial made of a semi-infinitely extended metal-dielectric multilayer. Red/green arrows represent $\mathrm{FF} / \mathrm{SH}$ photons radiated into the structure. (b) Real parts of the extraordinary [red (dashed-dotted line)] and ordinary [blue (solid line)] permittivities of the uniaxial anisotropic effective-medium model of a metal-dielectric stack with a metal fill factor $f=0.5$. The black (dashed) line is the RC angle $\theta_{\mathrm{RC}}$. the effective permittivity tensor $\boldsymbol{\varepsilon}=\varepsilon_{\mathrm{o}}(\hat{\mathbf{x}} \hat{\mathbf{x}}+\hat{\mathbf{y}} \hat{\mathbf{y}})+\varepsilon_{\mathrm{e}} \hat{\mathbf{z}} \hat{\mathbf{z}}$. This approximation has been tested several times in the past against full-wave approaches, such as the one based on the transfermatrix method, also for near-field excitations [12,13,32], and its limitations are now well understood. One may also resort to other methods, such as the nonlocal effective-medium model reported in Refs. [33,34]. However, the local model used here suffices because it provides a simple picture of the RCs' formation. Later in this section, we use a full-wave model of the discrete metal-dielectric structure that includes spatial dispersion and nonlocality due to the free-electron gas that validates the simple effective-medium approximation. In Sec. III, we use Bloch theory to highlight and to discuss strengths and limitations of the local homogenization.

In the local effective-medium model, ordinary and extraordinary permittivities $\varepsilon_{\mathrm{o}}=f \varepsilon_{\mathrm{m}}+(1-f) \varepsilon_{\mathrm{d}}$ and $\varepsilon_{\mathrm{e}}=$ $\left[f / \varepsilon_{\mathrm{m}}+(1-f) / \varepsilon_{\mathrm{d}}\right]^{-1}$ in that order, depend on the metal fill factor $f=t_{\mathrm{m}} /\left(t_{\mathrm{m}}+t_{\mathrm{d}}\right)$, where $t_{\mathrm{m}}$ and $t_{\mathrm{d}}$ are metal and dielectric layer thicknesses, respectively. We set $f=0.5$. The problem of a line dipole radiating in a linear, planar [11,13,35], or cylindrical [36] metal-dielectric stack has been studied extensively. In the planar geometry, it is straightforward to demonstrate that the two-dimensional scalar Green's function is singular when $\left(x-x^{\prime}\right)^{2} \varepsilon_{\mathrm{e}}+\left(z-z^{\prime}\right)^{2} \varepsilon_{\mathrm{o}}=0$ in a lossless medium, where $(x, z)$ and $\left(x^{\prime}, z^{\prime}\right)$ are observation and source points, respectively. For a lossy uniaxial crystal with $\operatorname{Re}\left[\varepsilon_{\mathrm{e}}\right]$ and $\operatorname{Re}\left[\varepsilon_{\mathrm{o}}\right]$ having opposite signs, the singularity is removed but manifests itself as a RC with a semiangle,

$$
\theta_{\mathrm{RC}}=\tan ^{-1}\left[\sqrt{-\operatorname{Re}\left(\varepsilon_{\mathrm{o}}\right) / \operatorname{Re}\left(\varepsilon_{\mathrm{e}}\right)}\right]
$$

evaluated with respect to the optical axis. A detailed vectorial description of hyperbolic media in terms of the Green's function may be found in Ref. [37]. In Fig. 1(b), we sum up the linear properties of the hyperbolic metamaterial described above by plotting the real parts of ordinary and extraordinary permittivities and the semiangle of the RC $\theta_{\mathrm{RC}}$. The RC reduces to a single volume plasmon polariton propagating along the optical axis $\left(\theta_{\mathrm{RC}}=0\right)$ when the ordinary permittivity is zero, i.e., when $\lambda=\lambda_{0}=405 \mathrm{~nm}$. At larger wavelengths, volume plasmon polaritons propagate in RCs with angle $\theta_{\mathrm{RC}}>0$.

We now consider the field emitted by an electric line dipole $\mathbf{p}=p_{x} \hat{\mathbf{x}} e^{-i \omega t}$ (infinitely extended in the $y$ direction) located in free space at a distance $h=5 \mathrm{~nm}$ from the semi-infinite substrate shown in Fig. 1(a). The distance $h$ affects only the transverse size and the strength of the volume plasmon polaritons without significantly altering the RC angles. A detailed discussion on the effects of dipole-substrate distance is found in Refs. [13,32]. We assume, for the sake of simplicity, that the substrate, whose effective parameters are summarized in Fig. 1(b), has a homogeneous instantaneous quadratic nonlinearity with nonzero susceptibility components $\chi_{x x x}^{(2)}=\chi_{z z z}^{(2)}=1 \mathrm{pm} / \mathrm{V}$. We stress that anisotropic models of the homogenized effective $\chi^{(2)}$ tensor, such as the one reported in Ref. [38], would lead to the same conclusions on the double RCs' formation, although conversion efficiency would be slightly different. The metamaterial scatters both FF and SH fields. Here we limit our attention to the fields radiated into the substrate region. One may safely assume that $\mathrm{SH}$ radiation remains a very small fraction of the FF 

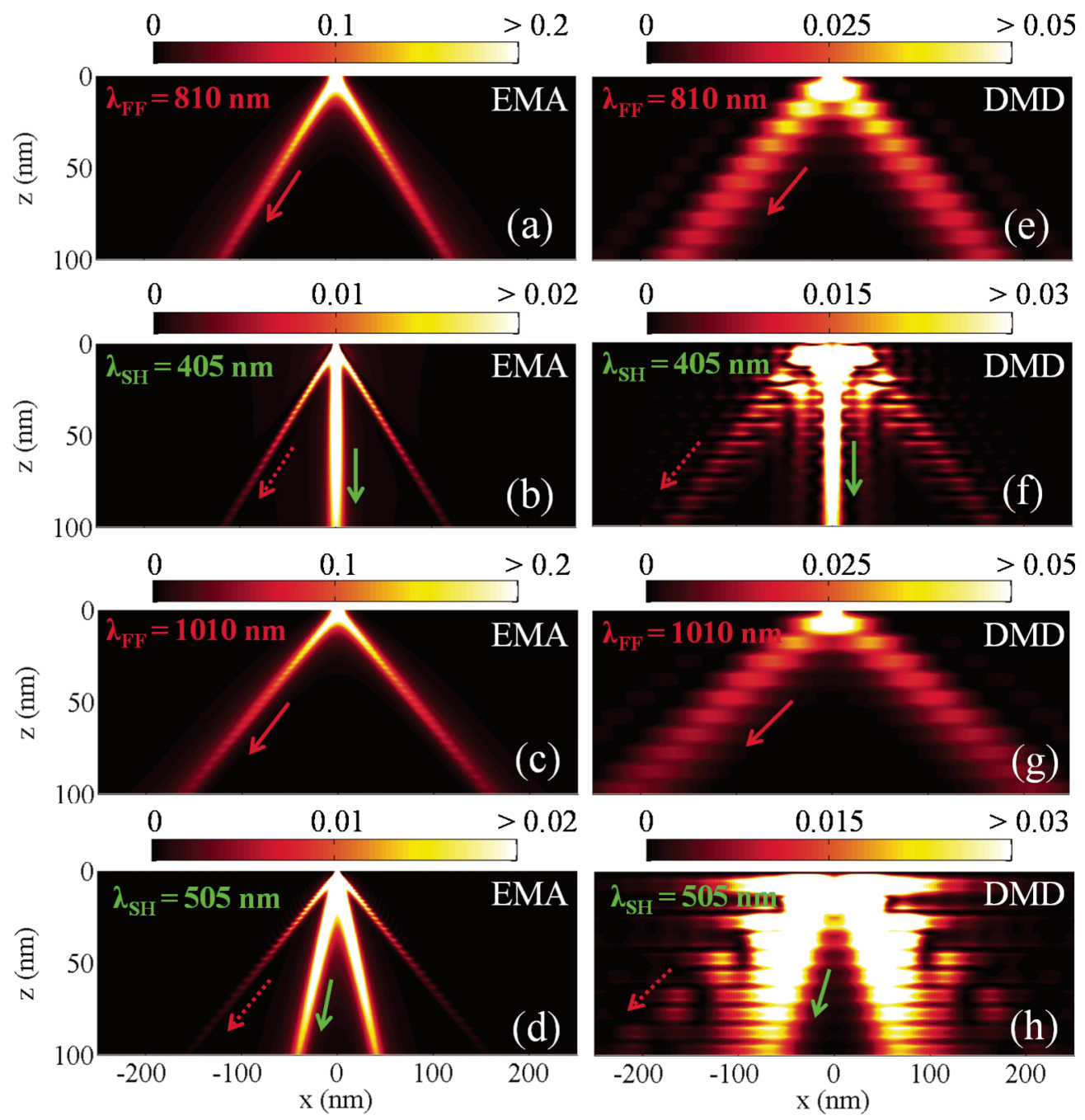

FIG. 2. (Color online) FF and SH magnetic-field intensity distributions generated by a dipole located 5 nm above the hyperbolic metamaterial. In (a)-(d), the field is calculated using the effective-medium approximation (EMA), whereas, in (e)-(h), the field is calculated for the lamellar discrete metal dielectric (DMD) geometry. In (a), the dipole emission wavelength is $\lambda_{\mathrm{FF}}=810 \mathrm{~nm}$. (b) The same as in (a) for the generated SH signal $\left(\lambda_{\mathrm{SH}}=405 \mathrm{~nm}\right)$. (c) The same as in (a) assuming $\lambda_{\mathrm{FF}}=1010 \mathrm{~nm}$. (d) The same as in (b) at $\lambda_{\mathrm{SH}}=505 \mathrm{~nm}$. (e)-(h) The same as in (a)-(d), respectively, calculated for the discrete geometry. The solid and dashed red arrows point in the same direction since they are associated with the FF and phase-locked SH RCs, respectively. The green arrows indicate the homogeneous SH RC. The color scale is linear and is compressed in order to show the field pattern inside the stack. The maximum is located in the volume of metamaterial just below the dipole.

field strength $\left|\mathbf{E}_{\mathrm{FF}}\right|$ so that the pump field propagates linearly and a perturbative approach may be undertaken. FF fields are calculated by solving the linear Helmholtz equation in the presence of the electric line dipole $\mathbf{p}$ [as illustrated in Fig. 1(a)] with a frequency-domain finite-element-method solver (RF module of COMSOL MULTIPHYSICS). The $\mathrm{SH}$ is found by solving the same linear problem at the $\mathrm{SH}$ frequency with sources provided by the solution of the FF problem using the finite-element method as explained below. We first tune the $\mathrm{SH}$ wavelength to satisfy $\theta_{\mathrm{RC}}\left(\lambda_{\mathrm{SH}}\right)=0$ so that $\lambda_{\mathrm{FF}}=2 \lambda_{\mathrm{SH}}=$ $2 \lambda_{0}=810 \mathrm{~nm}$. The volume plasmon polariton at the FF is tilted at $\theta_{\mathrm{RC}}\left(\lambda_{\mathrm{FF}}\right)=49^{\circ}$ with respect to the $z$ axis-Fig. 1(b). The magnetic-field intensity distribution $\left|H_{y}(x, z)\right|^{2}$ in the hyperbolic substrate at $\lambda_{\mathrm{FF}}=810 \mathrm{~nm}$-Fig. 2(a)-shows the propagation of subwavelength volume plasmon polaritons at $\theta_{\mathrm{RC}}\left(\lambda_{\mathrm{FF}}\right)= \pm 49^{\circ}$.
The source of the $\mathrm{SH}$ radiation is the electric volume current density $\mathbf{J}_{\mathrm{SH}}=-i \omega_{\mathrm{SH}} \varepsilon_{0}\left(\chi_{x x x}^{(2)} E_{\mathrm{FF}, x}^{2} \hat{\mathbf{x}}+\chi_{z z z}^{(2)} E_{\mathrm{FF}, z}^{2} \hat{\mathbf{z}}\right)$ induced by the FF signal in the substrate region, where $\omega_{\mathrm{SH}}$ is the $\mathrm{SH}$ angular frequency, $\varepsilon_{0}$ is the free-space permittivity, and $E_{\mathrm{FF}, x}$ and $E_{\mathrm{FF}, z}$ are the $\mathrm{FF}$ electric-field components in the $x$ and $z$ directions, respectively. In the undepleted pump approximation, the electromagnetic problem for $\mathrm{SH}$ light is described by the inhomogeneous wave equation $\nabla \times \nabla \times$ $\mathbf{E}_{\mathrm{SH}}-\omega_{\mathrm{SH}}^{2} / c^{2} \boldsymbol{\varepsilon}_{\mathrm{SH}} \cdot \mathbf{E}_{\mathrm{SH}}=i \omega_{\mathrm{SH}} \mu_{0} \mathbf{J}_{\mathrm{SH}}$, where $\mathbf{E}_{\mathrm{SH}}$ is the $\mathrm{SH}$ field, $c$ and $\mu_{0}$ are the speed of light and permeability of free space, respectively, $\boldsymbol{\varepsilon}_{\mathrm{SH}}$ is the permittivity tensor at the $\mathrm{SH}$ frequency, and the spatial dependence is implicit in $\mathbf{E}_{\mathrm{SH}}, \mathbf{J}_{\mathrm{SH}}$, and $\boldsymbol{\varepsilon}_{\mathrm{SH}}$. The solution for $\mathbf{E}_{\mathrm{SH}}$ is found numerically by solving the weak form of the inhomogeneous Helmholtz equation with a finite-element-based software (COMSOL MULTIPHYSICS). Note that this formulation can be applied to the anisotropic 
homogenized model and to the multilayer structure in Fig. 1. Even if the intensity of the current $\mathbf{J}_{\mathrm{SH}}$ peaks in the RC of the FF signal, i.e., at $\pm \theta_{\mathrm{RC}}\left(\lambda_{\mathrm{FF}}\right)$, the $\mathrm{SH}$ field also shows maxima in the directions $\pm \theta_{\mathrm{RC}}\left(\lambda_{\mathrm{SH}}\right)$, i.e., the $\mathrm{RC}$ at the $\mathrm{SH}$ wavelength. The generated $\mathrm{SH}$ radiation splits into two main RCs: (i) the phase-locked $\mathrm{SH}$ cone at $\pm \theta_{\mathrm{RC}}\left(\lambda_{\mathrm{FF}}\right)$ that overlaps with the $\mathrm{RCs}$ at the $\mathrm{FF}$ and (ii) the homogeneous cone generated in the volume of the hyperbolic metamaterial just below the dipole. The presence of a single homogenous cone is due to the fact that the absolute maximum of $\left|\mathbf{J}_{\mathrm{SH}}\right|$ occurs in the uppermost region of the metamaterial, i.e., the region closest to the dipole, because of the strong emitter near-field. The $\mathrm{SH}$ component in the homogeneous cone walks off the RC at the FF and freely propagates along the $\pm \theta_{\mathrm{RC}}\left(\lambda_{\mathrm{SH}}\right)$ directions. For $\lambda_{\mathrm{FF}}=810 \mathrm{~nm}$, the phase-locked cone is tilted by $\theta_{\mathrm{RC}}\left(\lambda_{\mathrm{FF}}\right) \approx 49^{\circ}$ with respect to the $z$ direction; the homogeneous cone $\pm \theta_{\mathrm{RC}}\left(\lambda_{\mathrm{SH}}\right)$ is emitted at $\theta_{\mathrm{RC}}\left(\lambda_{\mathrm{SH}}\right)=0^{\circ}$ since $\lambda_{\mathrm{SH}}=\lambda_{0}$. We note that, under TM-polarized light, a slab of stacked metal-dielectric layers with hyperbolic dispersion acts as a subwavelength imaging lens for SH light [39], thus, providing strong spectral isolation from the excitation signal. This concept will be illustrated in Sec. IV.

In Figs. 2(c) and 2(d), we report the case for $\lambda_{\mathrm{FF}}=$ $2 \lambda_{\mathrm{SH}}=1010 \mathrm{~nm}$. Now, the FF and phase-locked $\mathrm{SH}$ cones are tilted by $\theta_{\mathrm{RC}}\left(\lambda_{\mathrm{FF}}\right) \approx 57^{\circ}$; the homogeneous $\mathrm{SH}$ cone is found at $\theta_{\mathrm{RC}}\left(\lambda_{\mathrm{SH}}\right) \approx 22^{\circ}$ in accordance with Fig. 1(b). These results show that the theory of homogeneous and phase-locked $\mathrm{SH}$ generation, applied earlier to plane waves or beams propagating in regimes of negligible diffraction [1-5], may be extended to subwavelength volume plasmon polaritons propagating in RCs of hyperbolic metamaterials. Waves with spectral wave numbers propagating along the phase-locked $\mathrm{SH}$ (or FF) cone travel at the same phase and group velocities and attenuation rate of the FF. In contrast, waves with spectral wave numbers belonging to the homogeneous $\mathrm{SH}$ cone propagate with the phase and group velocities and attenuation rate of the hyperbolic medium at the $\mathrm{SH}$ frequency.

The anisotropic effective-medium model adopted above is valid strictly for metal-dielectric stacks with very small layer thicknesses. The homogenization model generally overstates the structure's ability to support propagation of large wave numbers. In fact, hyperbolicity of the dispersion relation is limited by layer thicknesses [12,13,32]. For these reasons, despite the simplicity of the effective-medium model, we also perform full-wave SH generation calculations by considering the discrete metal-dielectric stack geometry depicted in Fig. 1(a). We assume that a bulk nonlinearity is present only in the dielectric layers in the form: $\chi_{x x x}^{(2)}=\chi_{z z z}^{(2)}=$ $1 \mathrm{pm} / \mathrm{V}$ and that the second-harmonic contribution arising from the metal (surface and volume sources) is nonzero but, nevertheless, negligible. The magnetic-field intensity distributions $\left|H_{y}(x, z)\right|^{2}$ for the same FF/SH wavelengths as in Figs. $2(\mathrm{a})-2(\mathrm{~d})\left(\lambda_{\mathrm{FF}}=2 \lambda_{\mathrm{SH}}=2 \lambda_{0}=810\right.$ and $\lambda_{\mathrm{FF}}=2 \lambda_{\mathrm{SH}}=$ $1010 \mathrm{~nm}$ ) are now shown in Figs. 2(e)-2(h). The presence of volume plasmon polaritons propagating under $\mathrm{RCs}$, and more importantly, the phenomenology of double-resonance-cone formation in the $\mathrm{SH}$ radiation pattern, are preserved when one compares the results from the effective-medium model with those from the discrete lamellar geometry. Two RCs are clearly visible in the SH field distribution: homogeneous and phase-locked components. Despite the similarities, the models differ in several respects. For example, the RC angles are slightly larger, and the transverse size of the volume plasmon polaritons is wider for the discrete stack. In particular, we find that, when $\lambda_{\mathrm{FF}}=2 \lambda_{\mathrm{SH}}=2 \lambda_{0}=810 \mathrm{~nm}$, FF and phaselocked SH RCs are found at $56^{\circ}$ for the discrete case compared to $49^{\circ}$ for the homogenized hyperbolic model, whereas, the homogeneous SH RC is perpendicular to the interfaces in both models. When $\lambda_{\mathrm{FF}}=2 \lambda_{\mathrm{SH}}=1010 \mathrm{~nm}, \mathrm{FF}$ and phase-locked SH RCs are tilted at $62^{\circ}$ for the discrete stack compared to $57^{\circ}$ using the effective-medium model, whereas, the homogeneous $\mathrm{SH} \mathrm{RC}$ is tilted by $34^{\circ}$ for the discrete case compared to $22^{\circ}$ in the homogenized hyperbolic model.

\section{BLOCH THEORY ANALYSIS OF RESONANCE CONES: BEYOND THE EFFECTIVE-MEDIUM APPROXIMATION}

We further examine the differences between the homogeneous and the discrete models to gain insight into the physics of hyperbolic media by using Bloch theory for bulk plasmonic modes supported by the multilayer structure [13,29]. In Figs. 3(a) and 3(b), we plot the isofrequency diagrams of real and imaginary parts of the Bloch longitudinal wave number $k_{B, z}=\beta_{z}+i \alpha_{z}$ for two wavelengths: (i) $2 \lambda_{0}=810 \mathrm{~nm}$
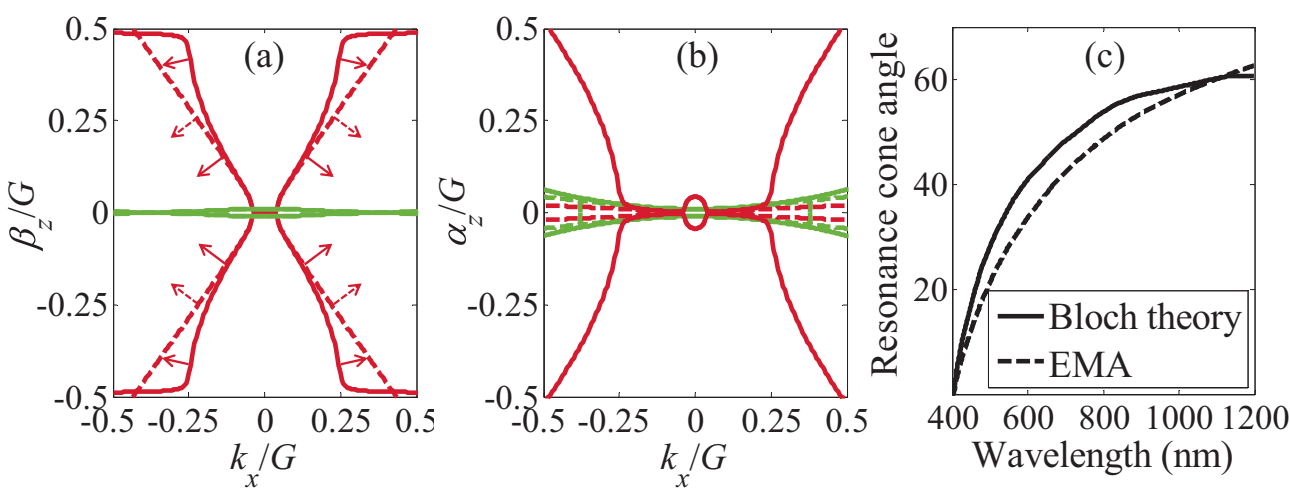

FIG. 3. (Color online) Isofrequency diagrams at $810 \mathrm{~nm}$ (red) and $405 \mathrm{~nm}$ (green) on the (a) $k_{x}-\beta_{z}$ and (b) $k_{x}-\alpha_{z}$ planes for the metal-dielectric multilayer described in Fig. 1(a). The solid lines refer to Bloch theory, and the dashed lines refer to the anisotropic effective-medium approximation (labeled EMA). The arrows indicate the direction of the group velocity; (c) RC angle with respect to the $z$ axis evaluated via Bloch theory, i.e., $\left\langle\theta_{\mathrm{BT}}\right\rangle$ and with the anisotropic effective-medium model, i.e., $\theta_{\mathrm{EMA}}$. 
(red curves) and (ii) $\lambda_{0}=405 \mathrm{~nm}$ (green curves), i.e., the $\mathrm{FF}$ and $\mathrm{SH}$ wavelengths of the radiation patterns shown in Figs. 2(a), 2(b), 2(e), and 2(f). In the representation of Figs. 3(a) and 3(b), $k_{x}$ is the transverse wave number, assumed purely real, of the spectral components emitted by the dipole, whereas, $k_{B, z}=\beta_{z}+i \alpha_{z}$ is the complex Bloch wave number, and $G=2 \pi /\left(t_{\mathrm{m}}+t_{\mathrm{d}}\right)$ is the magnitude of the reciprocal lattice vector. For comparison, the isofrequency diagrams obtained using the homogenized anisotropic dispersion relation $k_{x}^{2} / \varepsilon_{\mathrm{e}}+k_{z}^{2} / \varepsilon_{\mathrm{o}}=k^{2}$ are illustrated with dashed curves ( $k$ is the free-space wave number).

A significant deviation from the hyperbolic behavior predicted by the effective-medium approximation is observed at $810 \mathrm{~nm}$ [i.e., the difference between solid and dashed red curves in Figs. 3(a) and 3(b)]. The flattening of $\beta_{z}$ for high $k_{x}$ components and the associated increase in $\alpha_{z}$ are due to the modes approaching the edge of the first Brillouin zone. The effective-medium model is remarkably accurate at $\lambda_{0}=405 \mathrm{~nm}$ as suggested by the overlapping region of dashed and solid green curves in Figs. 3(a) and 3(b) and by looking at the virtually identical direction and transverse size of the homogeneous volume plasmon polaritons in the effective-medium approximation [Fig. 2(b)] and in the discrete geometry [Fig. 2(f)].

Isofrequency diagrams also contain information about the direction of the group velocity of each $k_{x}$ component. The group velocity $\nabla_{\mathbf{k}} \omega(\mathbf{k})$ is perpendicular to the frequency contours and points in the direction of increasing frequency $\omega$ [40]. This direction is evaluated as $\theta\left(k_{x}\right)=\tan ^{-1}\left(\partial \beta_{z} / \partial k_{x}\right)$. Inspecting first the diagrams related to the effective-medium model [dashed curves in Figs. 3(a) and 3(b)], we observe that the angle $\theta_{\mathrm{EMA}}\left(k_{x}\right)=\tan ^{-1}\left(\partial \beta_{z} / \partial k_{x}\right)$ is nearly constant varying $k_{x}$. In other words, the dashed arrows in Fig. 3(a), which indicate $\theta_{\mathrm{EMA}}\left(k_{x}\right)$ in the homogenized hyperbolic metamaterial, point in the same preferred direction regardless of the chosen $k_{x}$ component. We note that this preferred direction coincides with the RC angle [Eq. (1)], derived by examining the shape of the Green's function singularity. This result is expected because both definitions of the RC angle, one in the spatial frequency domain $\left[\theta_{\mathrm{EMA}}\left(k_{x}\right)\right]$ based on the group-velocity direction, the other in the real-space domain $\left(\theta_{\mathrm{RC}}\right)$ based on the Green's function, originate from the effective-medium approximation. Hence, one may surmise that $\theta_{\mathrm{EMA}}\left(k_{x}\right) \approx \theta_{\mathrm{RC}}$. In contrast, the group-velocity angle $\theta_{\mathrm{BT}}\left(k_{x}\right)=\tan ^{-1}\left(\partial \beta_{z} / \partial k_{x}\right)$ in the metal-dielectric multilayer modeled via Bloch theory is not constant. In fact, $\theta_{\mathrm{BT}}\left(k_{x}\right)$ is very close to $\theta_{\mathrm{RC}}$ only for small $k_{x}$ components $\left(\left|k_{x}\right| / G<0.1\right)$, becoming much larger than $\theta_{\mathrm{RC}}$ for $k_{x}$ components near and above the edge of the first Brillouin zone $\left(\left|k_{x}\right| / G>0.1\right)$. This may be inferred by looking at the solid red arrows in Fig. 3(a), which indicate the group-velocity direction in the periodic structure $\theta_{\mathrm{BT}}\left(k_{x}\right)$. A zeroth-order approximation of the $\mathrm{RC}$ angle in the stack is given by the weighted average,

$$
\left\langle\theta_{\mathrm{BT}}\right\rangle=\int_{0}^{\infty} \alpha_{z}^{-1}\left(k_{x}\right) \theta_{\mathrm{BT}}\left(k_{x}\right) d k_{x} / \int_{0}^{\infty} \alpha_{z}^{-1}\left(k_{x}\right) d k_{x},
$$

where the function $\alpha_{z}^{-1}\left(k_{x}\right)$ weighs the radiation angle $\theta_{\mathrm{BT}}\left(k_{x}\right)$ of each $k_{x}$ component with respect to its characteristic propagation distance (i.e., attenuation length) in the multilayer.

In Fig. 3(c), we show the wavelength dependence of the $\mathrm{RC}$ angle evaluated via the effective-medium approximation and Bloch theory, defined by $\theta_{\mathrm{EMA}}$ (dashed curve) and $\left\langle\theta_{\mathrm{BT}}\right\rangle$ (solid curve), respectively. It turns out that, in the wavelength range of $405-1010 \mathrm{~nm},\left\langle\theta_{\mathrm{BT}}\right\rangle>\theta_{\mathrm{EMA}}$. This result explains the discrepancies between radiation patterns evaluated with the two models at $810 \mathrm{~nm}$ [Figs. 2(a) and 2(e)] and the good agreement between the two models at $405 \mathrm{~nm}$ [Figs. 2(b) and 2(f)] where $\left\langle\theta_{\mathrm{BT}}\right\rangle \approx \theta_{\mathrm{EMA}}$.

\section{SECOND-HARMONIC IMAGING IN HYPERBOLIC DISPERSIVE MEDIA}

Subwavelength imaging through metal-dielectric stacks has been widely demonstrated in the linear regime [17-23,25,26]. Here we illustrate how the hyperbolic dispersion of the metal-dielectric stack described in Fig. 1(a), keeping the same geometry and materials reported in the previous sections, may be used to achieve subwavelength imaging and super-resolution for $\mathrm{SH}$ light. The effect is achieved by suppressing the phase-locked $\mathrm{SH}$ volume plasmon polariton and by exploiting only the homogeneous one. We consider two cylindrical nanoparticles of 5-nm diameters with nonzero quadratic nonlinearity and a dispersionless refractive index of 2 . We assume that the nonlinearity of the two nanocylinders is much larger than any nonlinearity within the multilayer stack, a reasonable assumption that makes only the two particles act like point dipoles emitting SH light. This assumption is necessary for the generated $\mathrm{SH}$ light to be channeled at the angle $\theta_{\mathrm{RC}}\left(\lambda_{\mathrm{SH}}\right)$ only and result in superresolution. The particles are separated by a subwavelength center-to-center distance of $50 \mathrm{~nm}$, and they are located in free space at a distance of $5 \mathrm{~nm}$ above the top layer (at $z=0$ ) of the multilayer stack. The setup is sketched in Fig. 4. The system is probed by a TM-polarized plane wave incident perpendicularly with respect to the stack. As reported in Sec. II, for an incident wavelength $\lambda_{\mathrm{FF}}=2 \lambda_{0}=810 \mathrm{~nm}$, the stack produces a $\mathrm{RC}$ at $\theta_{\mathrm{RC}}\left(\lambda_{\mathrm{FF}}\right) \approx 49^{\circ}$ at the FF. The homogeneous and phase-locked $\mathrm{RCs}$ for SH light occur at $0^{\circ}$ and $49^{\circ}$, respectively.

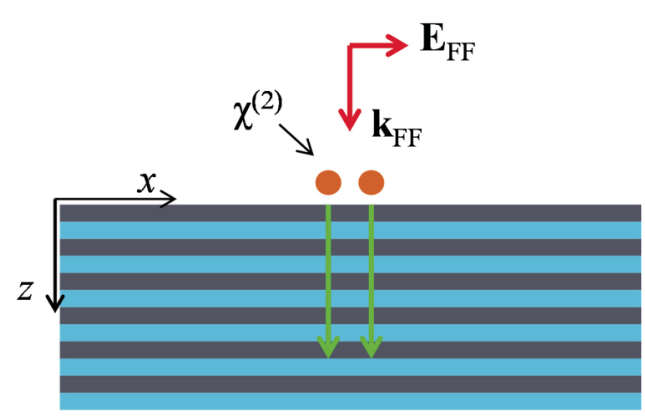

FIG. 4. (Color online) Sketch of the super-resolved near-field imaging at the $\mathrm{SH}$ wavelength. Two small particles with nonzero $\chi^{(2)}$ and interparticle distance of $50 \mathrm{~nm}$ are illuminated with a TM-polarized plane wave $\left(\lambda_{\mathrm{FF}}=810 \mathrm{~nm}\right)$. Second-harmonic light (represented by the two green arrows) creates a super-resolved image of the two particles. 

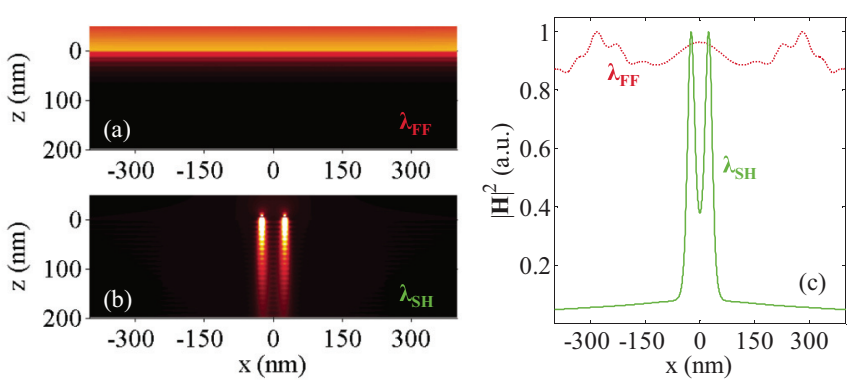

FIG. 5. (Color online) Magnetic-field intensity pattern at the (a) FF and (b) SH frequency for the setup in Fig. 1. (c) Cross section of (a) and (b) at $z=150 \mathrm{~nm}$.

In Fig. 5, we report the magnetic-field intensity pattern both at the FF and at the $\mathrm{SH}$ frequencies, where $\lambda_{\mathrm{SH}}=$ $\lambda_{\mathrm{FF}} / 2=405 \mathrm{~nm}$. The field at the FF [Fig. 5(a)] is only slightly perturbed by the presence of the two particles since the scattered field at this frequency is weaker than the illuminating plane wave. Instead, SH generated light is rather noticeable and is channeled into the homogeneous $\mathrm{SH}$ resonance cone, which travels straight down, perpendicular with respect to the interfaces [see Fig. 5(b)]. In this case, the phase-locked SH cone is not visible because the two particles exhibit very small scattering cross section at the FF and act mainly like point dipoles emitting SH light which is not blinded by the incident FF signal.

In Fig. 5(c), we also show the magnetic-field intensity at a section inside the stack $(z=150 \mathrm{~nm})$. Although the diameter of the two objects is small $\left(\sim \lambda_{\mathrm{FF}} / 160\right)$ and the separation is extremely subwavelength with respect to the incident wavelength $\left(\sim \lambda_{\mathrm{FF}} / 16\right)$, the image at the SH wavelength clearly shows the presence of the two objects at a distance of $150 \mathrm{~nm}$ within the hyperbolic metamaterial. We note that, although super-resolution is limited by the cutoff of evanescent waves, the maximum object-to-image distance at which super-resolution is observable is only limited by losses in the stack.

\section{CONCLUSIONS}

To summarize, we have discussed the formation of double $\mathrm{RCs}$ in nonlinear dispersive hyperbolic metamaterials. We have demonstrated that a quadratic nonlinearity in metaldielectric multilayers generates two subwavelength volume plasmon polaritons: one associated with the homogeneous $\mathrm{SH}$ component that propagates in a small-angle RC, the other phase locked under the larger-angle $\mathrm{RC}$ at the FF. The differences between the effective-medium model and the metal-dielectric stack consist of a wider volume plasmon cross section and larger RC angles in the metal-dielectric geometry due to the cutoff of high $k_{x}$ components near and above the first Brillouin zone. SH double RCs may drastically improve the subwavelength imaging abilities of metamaterial-based lenses thanks to the large spectral separation between the excitation and the observed signals. We have shown a representative case in which super-resolution is attained by exploiting the SH light channeled into the homogeneous volume plasmon polariton. Double RCs are predicted in the radiation patterns at second- and higher-order harmonic wavelengths in any anisotropic medium with hyperbolic dispersion. Although we have considered planar metal-dielectric multilayers, similar phenomenology is also expected in other implementations, such as nanowire metamaterials [26,41] and multilayer graphene structures $[42,43]$.

\section{ACKNOWLEDGMENTS}

This research was performed while authors D. de Ceglia, M. A. Vincenti, and J. W. Haus held National Research Council Research Associateship awards at the U.S. Army Aviation and Missile Research Development and Engineering Center.
[1] J. A. Armstrong, N. Bloembergen, J. Ducuing, and P. S. Pershan, Phys. Rev. 127, 1918 (1962).

[2] N. Bloembergen and P. S. Pershan, Phys. Rev. 128, 606 (1962).

[3] L. D. Noordam, H. J. Bakker, M. P. de Boer, and H. B. van Linden van den Heuvell, Opt. Lett. 16, 971 (1991).

[4] V. Roppo, M. Centini, C. Sibilia, M. Bertolotti, D. de Ceglia, M. Scalora, N. Akozbek, M. J. Bloemer, J. W. Haus, O. G. Kosareva, and V. P. Kandidov, Phys. Rev. A 76, 033829 (2007).

[5] M. Centini, V. Roppo, E. Fazio, F. Pettazzi, C. Sibilia, J. W. Haus, J. V. Foreman, N. Akozbek, M. J. Bloemer, and M. Scalora, Phys. Rev. Lett. 101, 113905 (2008).

[6] C. L. Cortes, W. Newman, S. Molesky, and Z. Jacob, J. Opt. 14, 063001 (2012).

[7] M. Noginov, M. Lapine, V. Podolskiy, and Y. Kivshar, Opt. Express 21, 14895 (2013).

[8] A. Poddubny, I. Iorsh, P. Belov, and Y. Kivshar, Nat. Photonics 7, 948 (2013).

[9] D. R. Smith, D. Schurig, J. J. Mock, P. Kolinko, and P. Rye, Appl. Phys. Lett. 84, 2244 (2004).

[10] D. R. Smith and D. Schurig, Phys. Rev. Lett. 90, 077405 (2003).
[11] L. B. Felsen and N. Marcuvitz, Radiation and Scattering of Waves (Prentice-Hall, Upper Saddle River, NJ, 1973).

[12] O. Kidwai, S. V. Zhukovsky, and J. E. Sipe, Opt. Lett. 36, 2530 (2011).

[13] C. Guclu, S. Campione, and F. Capolino, Phys. Rev. B 86, 205130 (2012).

[14] O. Shramkova and A. Schuchinsky, Int. J. RF Microwave Comput.-Aided Eng. 22, 469 (2012).

[15] D. Aronovich and G. Bartal, Opt. Lett. 38, 413 (2013).

[16] Z. Jacob, L. V. Alekseyev, and E. Narimanov, Opt. Express 14, 8247 (2006).

[17] S. A. Ramakrishna, J. B. Pendry, M. C. K. Wiltshire, and W. J. Stewart, J. Mod. Opt. 50, 1419 (2003).

[18] A. Salandrino and N. Engheta, Phys. Rev. B 74, 075103 (2006).

[19] M. Scalora, G. D’Aguanno, N. Mattiucci, M. J. Bloemer, D. de Ceglia, M. Centini, A. Mandatori, C. Sibilia, N. Akozbek, M. G. Cappeddu, M. Fowler, and J. W. Haus, Opt. Express 15, 508 (2007). 
[20] D. de Ceglia, M. Vincenti, M. Cappeddu, M. Centini, N. Akozbek, A. D’Orazio, J. Haus, M. Bloemer, and M. Scalora, Phys. Rev. A 77, 033848 (2008).

[21] P. A. Belov and Y. Hao, Phys. Rev. B 73, 113110 (2006).

[22] P. Belov, in Active Plasmonics and Tuneable Plasmonic Metamaterials, edited by A. V. Zayats and S. Maier (Wiley, Hoboken, NJ, 2013), p. 261.

[23] R. Kotyński and T. Stefaniuk, J. Opt. A: Pure Appl. Opt. 11, 015001 (2009).

[24] M. A. Vincenti, D. de Ceglia, V. Rondinone, A. Ladisa, A. D’Orazio, M. J. Bloemer, and M. Scalora, Phys. Rev. A 80, 053807 (2009).

[25] J. Bénédicto, E. Centeno, and A. Moreau, Opt. Lett. 37, 4786 (2012).

[26] C. R. Simovski, P. A. Belov, A. V. Atrashchenko, and Y. S. Kivshar, Adv. Mater. 24, 4229 (2012).

[27] R. K. Fisher and R. W. Gould, Phys. Rev. Lett. 22, 1093 (1969).

[28] S. Ishii, A. V. Kildishev, E. Narimanov, V. M. Shalaev, and V. P. Drachev, Laser Photonics Rev. 7, 265 (2013).

[29] B. Wood, J. B. Pendry, and D. P. Tsai, Phys. Rev. B 74, 115116 (2006).

[30] W. D. Newman, C. L. Cortes, and Z. Jacob, J. Opt. Soc. Am. B 30, 766 (2013).

[31] E. D. Palik, Handbook of Optical Constants of Solids (Academic, New York, 1985).
[32] O. Kidwai, S. V. Zhukovsky, and J. E. Sipe, Phys. Rev. A 85, 053842 (2012).

[33] A. V. Chebykin, A. A. Orlov, A. V. Vozianova, S. I. Maslovski, Y. S. Kivshar, and P. A. Belov, Phys. Rev. B 84, 115438 (2011).

[34] W. Yan, M. Wubs, and N. A. Mortensen, Phys. Rev. B 86, 205429 (2012).

[35] X. Ni, G. V. Naik, A. V. Kildishev, Y. Barnakov, A. Boltasseva, and V. M. Shalaev, Appl. Phys. B 103, 553 (2011).

[36] H. Liu and K. J. Webb, Opt. Lett. 36, 379 (2011).

[37] A. S. Potemkin, A. N. Poddubny, P. A. Belov, and Y. S. Kivshar, Phys. Rev. A 86, 023848 (2012).

[38] R. W. Boyd and J. E. Sipe, J. Opt. Soc. Am. B 11, 297 (1994).

[39] A. V. Zayats and D. Richards, Nano-Optics and Near-Field Optical Microscopy (Artech House, Norwood, MA, 2009).

[40] J. D. Joannopoulos, S. G. Johnson, J. N. Winn, and R. D. Meade, Photonic Crystals: Molding the Flow of Light (Princeton University Press, Princeton, 2011).

[41] J. Elser, R. Wangberg, V. A. Podolskiy, and E. E. Narimanov, Appl. Phys. Lett. 89, 261102 (2006).

[42] I. V. Iorsh, I. S. Mukhin, I. V. Shadrivov, P. A. Belov, and Y. S. Kivshar, Phys. Rev. B 87, 075416 (2013).

[43] M. A. K. Othman, C. Guclu, and F. Capolino, Opt. Express 21, 7614 (2013). 